\title{
Microscopic quantum theory of spatially resolved photoluminescence in semiconductor quantum structures
}

\author{
G. Pistone, ${ }^{\text {a) }}$ S. Savasta, O. Di Stefano, and R. Girlanda \\ Istituto Nazionale per la Fisica della Materia INFM and Dipartimento di Fisica della Materia e Tecnologie \\ Fisiche Avanzate, Università di Messina, Salita Sperone 31, I-98166 Messina, Italy
}

\begin{abstract}
We present a microscopic analysis of spatially resolved photoluminescence and photoluminescence excitation spectroscopy in semiconductor quantum structures. Such theoretical and numerical framework provides a general basis for the description of spectroscopic imaging in which the excitation and detection energies and spatial positions can all independently be scanned. The numerical results clarify the impact of the near-field optical setup on the obtained images and resolutions. [DOI: 10.1063/1.1711184]
\end{abstract}

In recent years, measurements based on spatially resolved photoluminescence (PL) provided direct information on the spatial and energy distribution of light emitting nanometric centers of semiconductor quantum structures, thus opening a rich area of physics involving spatially resolved quantum systems in a complex solid state environment. ${ }^{1-6}$ In particular, near-field optical microscopy and spectroscopy can detect the optical characteristics of individual quantum dots (QDs) among, e.g., a high-density ensemble of naturally formed QDs, ${ }^{7}$ whereas usual far-field methods provide only ensemble-averaged properties. Detailed simulations of Zimmermann, Runge, and Savona ${ }^{8,9}$ have clarified many aspects of the intrigued nonequilibrium dynamics giving rise to photoluminescence spectra in these quantum structures. However, theoretical simulations of near-field imaging spectroscopy of semiconductor quantum structures focus on calculations of local absorption. ${ }^{10-13}$ In contrast, as a matter of fact, almost all experimental images are obtained from PL measurements. Here we present a microscopic theory of spatially resolved photoluminescence in quantum structures that includes both light quantization (essential to describe radiative recombination) and phonon scattering. The theory also includes the description of spatially confined excitation (illumination mode) and/or detection (collection mode). It is worth noting that we are faced with a strictly nonequilibrium problem. Nonequilibrium here arising from both radiative recombination (preventing full thermalization) and from the local nature of the excitation source (in the illuminationmode setup). We are also faced with the problem of the differences between illumination $(I)$ and collection $(C)$ scanning near-field optical microscopy (SNOM) instruments. The equivalence between these two working modes has been established on the basis of the reciprocity theorem for electromagnetic fields. ${ }^{14}$ However, this theorem holds for linear and passive media. While semiconductor structures, at low excitation densities, show a linear behavior, phonon scattering and radiative recombination prevent them from being passive.

The positive frequency components of the operator de-

\footnotetext{
a) Author to whom correspondence should be addressed; electronic mail: pistone@unime.it
}

scribing the signal that can be detected by a general nearfield setup can be expressed as ${ }^{15} \hat{S}_{t}^{+}=\hat{A}_{\mathrm{bg}}^{+}+\hat{S}^{+}$, where $\hat{A}_{\mathrm{bg}}^{+}$is the elastic background signal (largely uniform along the $x-y$ plane) proportional to the input electric-field operator, and $\hat{S}^{+}$is related to the sample polarization density operator $\hat{\mathbf{P}}^{+}(\mathbf{r}): \hat{S}^{+}=\mathcal{A} \int d \mathbf{r} \hat{\mathbf{P}}^{+}(\mathbf{r}) \cdot \mathbf{E}_{C}(\mathbf{r})$, where $\mathcal{A}$ is a complex constant and $\mathbf{E}_{C}(\mathbf{r})$ is the signal mode delivered by the tip. Photoluminescence can be defined as the incoherent part of the emitted light intensity. ${ }^{16}$ The PL that can be measured by a photodetector after the collection setup (broadband detection) is proportional to $I_{\mathrm{PL}}=I-I_{\mathrm{coh}}=\left\langle\hat{S}^{-}(\tau) \hat{S}^{+}(\tau)\right\rangle$ $-\left|\left\langle\hat{S}^{+}(\tau)\right\rangle\right|^{2}$. Analogously, the steady-state spectrum of incoherent light emitted by the semiconductor quantum structure and detected by the SNOM setup can be expressed as

$$
\mathcal{I}\left(\omega_{C}\right)=\frac{1}{\pi} \int_{0}^{\infty} d \tau\left\langle\hat{S}^{-}(0) \hat{S}^{+}(\tau)\right\rangle e^{i \omega_{C} \tau} .
$$

The interband polarization density operator can be expressed in terms of exciton operators as $\hat{\mathbf{P}}^{+}(\mathbf{r})=\boldsymbol{\mu}_{\mathrm{eh}} f(z) \Psi_{\alpha}^{\mathrm{eh}}(\rho$ $=0, \mathbf{R}) \hat{B}_{\alpha}$, where $e$ and $h$ are appropriate sets of quantum numbers which label the carrier states involved in the optical transition. The operator $\hat{B}_{\alpha}^{\dagger}$ creates an exciton state (one electron-hole pair) $\hat{B}_{\alpha}^{\dagger}|0\rangle \equiv\left|E_{1, \alpha}\right\rangle$ with energy $\omega_{1, \alpha}$. $\Psi_{\alpha}^{\mathrm{eh}}(\rho, \mathbf{R})$ is the exciton wave function with $\rho$ indicating the relative in-plane electron-hole eh coordinate and $\mathbf{R}$ describes the center of mass motion, while $f(z)=u_{e}(z) u_{h}(z)$ is the product of the electron and hole envelope functions along the confinement direction. If the disorder induced broadening is small compared to the exciton binding energy, only the lowest bound state $1 s$ at the fundamental sublevel transition has to be considered and the exciton wave function can be factorized as $\Psi_{\alpha}^{\mathrm{eh}}(\rho, \mathbf{R})=\phi_{1 s}(\rho) \psi_{\alpha}(\mathbf{R}) .{ }^{9}$ In the low-excitation regime the relation $\hat{B}_{\alpha} \simeq|0\rangle\left\langle E_{1, \alpha}\right|$ can be assumed and it can be shown that the operators $\hat{B}_{\alpha}$ behave as Boson operators. ${ }^{17}$ Within these assumptions, the Hamiltonian determining the dynamics of the semiconductor system is given by the three contributions: the bare excitonic Hamiltonian of the semiconductor system, the interaction Hamiltonian of the semiconductor with the quantum (possibly inhomogeneous) light field and the interaction Hamiltonian of excitons with the phonon bath determining inelastic scattering and dephasing. ${ }^{9}$ The kinetic equation for diagonal terms of the exciton den- 
sity matrix $N_{\alpha}=\left\langle\hat{B}_{\alpha}^{\dagger} \hat{B}_{\alpha}\right\rangle$ can be derived starting from the Heisenberg equation of motion for the exciton operators under the influence of $H$. The main approximations are the neglect of possible coherent phonon states and of memory effects induced by the photon and phonon fields. The result is

$$
\partial_{t} N_{\alpha}=G_{\alpha}\left(\omega_{I}\right)+\sum_{\beta} \gamma_{\alpha \leftarrow \beta} N_{\beta}-2 \Gamma_{\alpha} N_{\alpha},
$$

where the resulting phonon-assisted scattering rates $\gamma_{\beta \leftarrow \alpha}$ are given by $\gamma_{\beta \leftarrow \alpha}=\left(2 \pi / \hbar^{2}\right) \Sigma_{\mathbf{q}}\left|t_{\alpha, \beta}^{\mathbf{q}}\right|^{2}\left[n\left(\omega_{\mathbf{q}}\right) \delta_{\alpha, \beta, \mathbf{q}}^{+}+(1\right.$ $\left.\left.+n\left(\omega_{\mathbf{q}}\right)\right) \delta_{\alpha, \beta, \mathbf{q}}^{-}\right], n\left(\omega_{\mathbf{q}}\right)$ being the thermal phonon occupation functions and $\delta_{\alpha, \beta, \mathbf{q}}^{ \pm}=\delta\left(\omega_{\alpha}-\omega_{\beta} \pm \omega_{\mathbf{q}}\right)$. The scattering matrix elements $t_{\alpha, \beta}^{\mathbf{q}}$ depend on the lattice deformation potentials and the overlap between the exciton states. ${ }^{9} 2 \Gamma_{\alpha}$ $=r_{\alpha}+\Sigma_{\beta} \gamma_{\beta \leftarrow \alpha}$ is the total out-scattering rate, $r_{\alpha}$ being the rate for radiative recombination proportional to the exciton oscillator strength: $r_{\alpha}=r_{0}\left|\int d^{2} \mathbf{R} \psi_{\alpha}(\mathbf{R})\right|^{2}$ (we assume that the tip-sample interaction does not alter the radiative decay rates). In the above equation the generation term depends on the spatial overlap between the illuminating field $E_{I}(\mathbf{r})$ and the exciton wave functions corresponding to exciton levels resonant with the input light: $G_{\alpha}\left(\mathbf{R}_{I}\right)=r_{0}\left|o_{\alpha}^{I}\left(\mathbf{R}_{I}\right)\right|^{2} \mathcal{L}_{\alpha}\left(\omega_{I}\right)$ with $\pi \mathcal{L}_{\alpha}(\omega)=\Gamma /\left[\left(\omega-\omega_{\alpha}\right)^{2}+\Gamma^{2}\right]$ and $o_{\alpha}^{I}\left(\mathbf{R}_{I}\right)$ $=\int d^{2} \mathbf{R} \widetilde{E}_{I}(\mathbf{R}) \psi_{\alpha}(\mathbf{R})$ where $\widetilde{E}_{I}(\mathbf{R})=\int E_{I}(\mathbf{r}) f(z) d z$. In the subsequent numerical calculations concerning the illumination mode we will assume an input light field with a given Gaussian profile centered around the tip position $\mathbf{R}_{I}$ : $\widetilde{E}_{I}(\mathbf{R})=E_{I}^{0} g\left(\mathbf{R}-\mathbf{R}_{I}\right)$. In this case the generation term becomes function of the beam position and shape (spatial resolution).

Once the exciton densities have been derived, the frequency integrated PL can be readily obtained. It results in $\mathcal{I}\left(\mathbf{R}_{C}\right)=r_{0} \Sigma_{\alpha}\left|o_{\alpha}^{C}\left(\mathbf{R}_{C}\right)\right|^{2} N_{\alpha}$, where $o_{\alpha}^{C}$, analogously to $o_{\alpha}^{I}$, contains the overlap of the exciton wave functions with the signal mode $\widetilde{E}_{C}(\mathbf{R})$ delivered by the tip (collection mode). According to the quantum regression theorem, $\left\langle\hat{S}^{-}(0) \hat{S}^{+}(\tau)\right\rangle$ has the same dynamics of $\left\langle\hat{S}^{+}(\tau)\right\rangle$ (proportional to the exciton operator), but with $\left\langle\hat{S}^{-}(0) \hat{S}^{+}(0)\right\rangle$ as initial condition. Following this procedure we obtain $\mathcal{I}\left(\mathbf{R}_{C}, \omega_{C}\right)=r_{0} \Sigma_{\alpha}\left|o_{\alpha}^{C}\left(\mathbf{R}_{C}\right)\right|^{2} \mathcal{L}_{\alpha}\left(\omega_{C}\right) N_{\alpha}$.

We apply the above developed theoretical scheme to analyze the spatially resolved light emission of a QD system arising from interface fluctuations of GaAs QWs. The effective disordered potential felt by $1 s$ excitons used in our simulations is obtained summing up two different contributions, both modeled as a zero mean, Gauss distributed and spatially correlated process. We used $\xi=16 \mathrm{~nm}$ (the correlation length), $v_{0}=1.5 \mathrm{meV}$ (the width of the energy distribution); and $\xi=8 \mathrm{~nm}, v_{0}=0.5 \mathrm{meV}$, respectively.

Figure 1(a) displays the specific realization of the effective disordered potential used for all the calculations. Figures 1(b) -1(d) show energy-integrated PL images obtained after uniform illumination of the sample at energy $\omega_{I}=1 \mathrm{meV}$ (the zero of energy is fixed at the energy of the $1 s$ exciton in absence of disorder) and collecting locally the emitted light [ $C$ mode with spatial resolution full width at half maximum $(\mathrm{FWHM})=47 \mathrm{~nm}]$. It is worth noting that the energyintegrated excitonic local density of states does not depend on position. So the observed structures are a direct consequence of the increasing ratio between radiative and nonra-
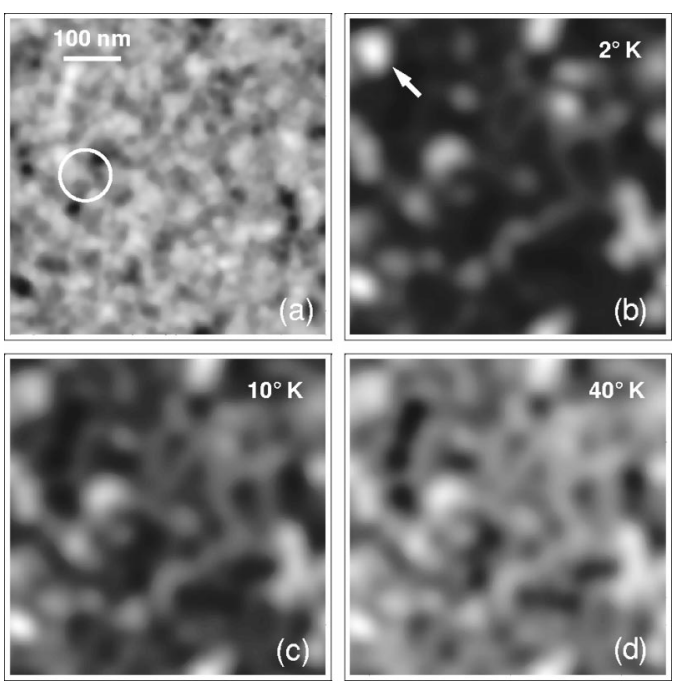

FIG. 1. (a). Specific realization of disorder potential; (b)-(d) energyintegrated PL images obtained after uniform illumination of the sample at energy $\omega_{I}=1 \mathrm{meV}$ and collecting locally the emitted light.

diative scattering rates for exciton states localized at the potential minima [compare, e.g., images 1(a) and 1(c)]. As the temperature of the structure is lowered, a transition from a broad and fairly continuous PL to an intense set of few spatially localized luminescence centers can be observed. Another interesting feature is the nonmonotonous brightness of some luminescence centers when the temperature is increased [see, e.g., the location indicated by an arrow in image 1(b)], due to excitons which can overcome shallow local minima by thermal activation and fall down in still deeper states. $^{8}$

In Fig. 2 we report the emission intensity as a function of both the illumination and collection energy for the three main working modes of the SNOM: I (local illumination, far-field collection), $C$ (far-field illumination, local collection) and $I-C$ mode (both local illumination and collection). The tip (a spatial resolution of FWHM=94 nm is assumed) for local illumination and/or detection is centered at the po-

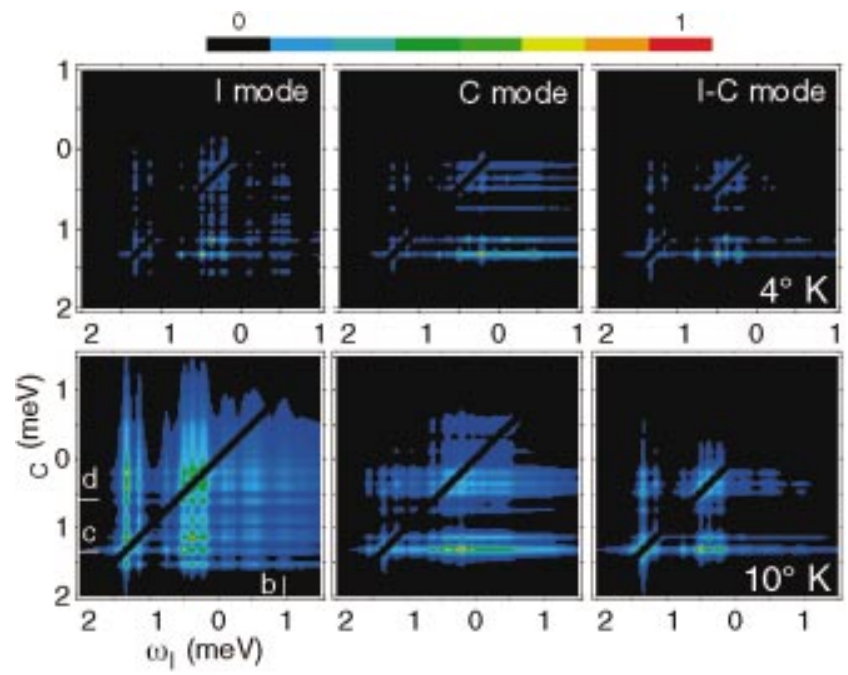

FIG. 2. (Color) Emission intensity as a function of both the illumination and collection energy for the three main working modes of the SNOM. A small region along the diagonal $\left(\omega_{C}=\omega_{I}\right)$ containing strong elastic contributions is canceled. 

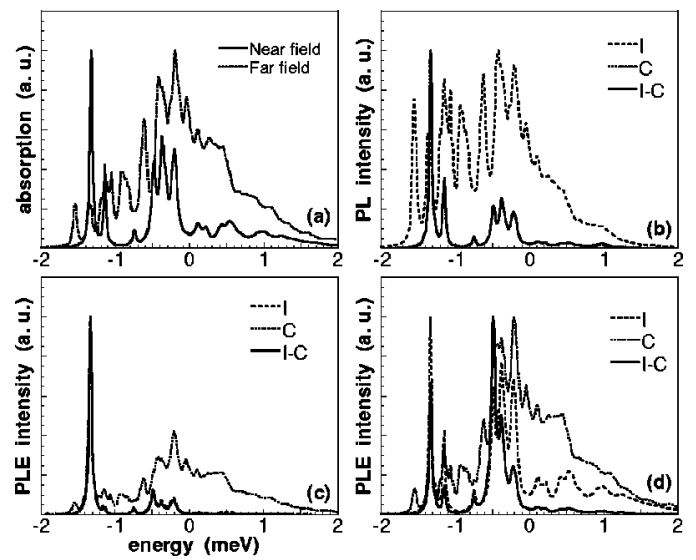

FIG. 3. (a) Far-field spectrum and local absorption spectrum calculated centering the tip at the position indicated by a circle in Fig. 1(a). (b),(c),(d) Line scans (PL and PLE spectra) indicated by the letters (b,c,d) in Fig. 2 for the three different working modes $\left(T=10^{0} \mathrm{~K}\right)$.

sition indicated by a circle in Fig. 1(a). Figure 2 clearly shows the impact that the specific working mode of the instrument can have on the obtained results. The $I$ - $C$ images are the most selective and show a series of discrete peaks that merge into quasicontinua (corresponding to less localized exciton states) at higher energy, whereas the $I$ mode is selective only for photoluminescence excitation (PLE) measurements ( $\omega_{C}$ fixed, $\omega_{I}$ tuned). On the contrary, the $C$ mode shows good selectivity for PL measurements $\left(\omega_{C}\right.$ tuned, $\omega_{I}$ fixed), being of poor selectivity for PLE. This picture can be better pointed out by looking at the sections of these grayscale plots shown in Fig. 3. Panel 3(a) displays for reference the far-field and local absorption spectra. Figure 3(b) shows that the $C$ and $I-C$ modes give the same results when the sample is excited at quite a high energy and $\omega_{C}$ is tuned. Also, the $I$ and $I$ - $C$ modes almost coincide [see Fig. 3(c)] when $\omega_{C}$ is fixed at a low energy absorption peak. However, Fig. 3(d) shows that in other circumstances the three working modes produce different results. It is also interesting to notice the differences in the peaks height between PLE and local absorption spectra. The strong nonequivalence between different SNOM kinds, here observed, is consequence (and at the same time a direct proof) of the nonlocal nature of the incoherent energy transfer between different regions of the sample involving length scales significantly larger than
SNOM spatial resolutions (even in presence of well defined dots). Further evidence is given by the strong differences between the calculated local absorption and emission spectra in some working mode. Finally, we note the thermal activation of light up-conversion due to phonon-assisted exciton scattering clearly visible in the lower panels of Fig. 2.

In summary we have presented a microscopic quantum theory of spatially resolved photoluminescence in quantum wells with interface fluctuations. The theory establishes a clear functionality of the different SNOM kinds when applied to the analysis of semiconductor quantum structures. Moreover, this formulation permits one to model PL excitation spectroscopy in which the excitation and detection energies and spatial positions can all independently be scanned. The numerical results here presented constitute an intriguing example of the impact of sample temperature, exciton localization, and microscope setup in the formation of subwavelength-resolution images.

${ }^{1}$ H. F. Hess, E. Betzig, T. D. Harris, L. N. Pfeiffer, and K. W. West, Science 264, 1740 (1994).

${ }^{2}$ D. Gammon, E. S. Snow, and D. S. Katzer, Appl. Phys. Lett. 67, 2391 (1995).

${ }^{3}$ D. Gammon, E. S. Snow, B. V. Shanabrook, D. S. Katzer, and D. Park, Phys. Rev. Lett. 76, 3005 (1996); Science 273, 87 (1996).

${ }^{4}$ Q. Wu, R. D. Grober, D. Gammon, and D. S. Katzer, Phys. Rev. Lett. 83, 2652 (1999).

${ }^{5}$ J. Hasen, L. N. Pfeiffer, A. Pinczuk, S. He, K. W. West, and B. S. Dennis, Nature (London) 390, 54 (1997).

${ }^{6}$ S.-K. Eah, W. Jhe, and Y. Arakawa, Appl. Phys. Lett. 80, 2779 (2002).

${ }^{7}$ A. Zrenner, L. V. Butov, M. Hagn, G. Abstreiter, G. Böhm, and G. Weimann, Phys. Rev. Lett. 72, 3382 (1994).

${ }^{8}$ R. Zimmermann and E. Runge, Phys. Status Solidi A 164, 511 (1997).

${ }^{9}$ R. Zimmermann, E. Runge, and S. Savona, in Quantum Coherence, Correlation and Decoherence in Semiconductor Nanostructures, edited by $\mathrm{T}$. Takagahara (Elsevier, New York, 2003), pp. 89-165.

${ }^{10}$ O. Mauritz, G. Goldoni, F. Rossi, and E. Molinari, Phys. Rev. Lett. 82, 847 (1999).

${ }^{11}$ O. Di Stefano, S. Savasta, G. Martino, and R. Girlanda, Appl. Phys. Lett. 77, 2804 (2000).

${ }^{12}$ O. Di Stefano, S. Savasta, and R. Girlanda, J. Appl. Phys. 91, 2302 (2002).

${ }^{13}$ G. Pistone, S. Savasta, O. Di Stefano, and R. Girlanda, Phys. Rev. B 67, 153305 (2003).

${ }^{14}$ E. R. Mendez, J.-J. Greffet, and R. Carminati, Opt. Commun. 142, 7 (1997).

${ }^{15}$ J.-J. Greffet and R. Carminati, Prog. Surf. Sci. 56, 133 (1997).

${ }^{16}$ M. Pieruccini, S. Savasta, R. Girlanda, R. C. Iotti, and F. Rossi, Appl. Phys. Lett. 83, 2480 (2003).

${ }^{17}$ V. M. Axt, K. Victor, and A. Stahl, Phys. Rev. B 53, 7244 (1996). 